\title{
Cationic amphiphiles bearing a diacetylenic function in the headgroup: aggregative properties and polymerization
}

Alessandro Mauceri, $\$+$ Luisa Giansanti, ‘ Donatella Capitani, ${ }^{\dagger}$ Anatoly Sobolev, ${ }^{+}$Luciano Galantini,§ Mauro Bassetti," Maria Grazia Nemi," Denise Gradella Villalva, $\$+$ Sara Battista, " Giovanna Mancini ${ }^{+*}$

§Dipartimento di Chimica, Università di Roma "Sapienza", P.le A. Moro 5, 00185 Roma (Italy); ”Dipartimento di Scienze Fisiche e Chimiche, Università degli Studi dell'Aquila, Via Vetoio 10, 67010 Coppito (Italy); ${ }^{+}$CNR - Istituto per i Sistemi Biologici, Via Salaria km 29.300, 00016 Monterotondo Scalo, RM, Italy; "CNR, Istituto per i Sistemi Biologici c/o Dipartimento di Chimica, Università di Roma "Sapienza", P.le A. Moro 5, 00185 Roma (Italy).

Supplementary Information

SI1. CONTIN distributions of CD1, CD2 and CD3 reported as an example

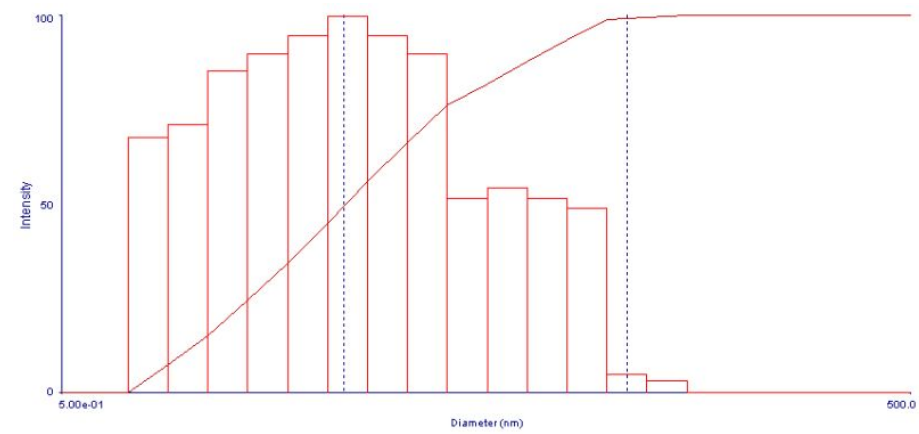

\begin{tabular}{|rrr|rrr|rrr|}
\hline$d$ & $G(d)$ & $C(d)$ & $d$ & $G(d)$ & $C(d)$ & $d$ & $G(d)$ & $C(d)$ \\
\hline 1.00 & 68 & 7 & 35.47 & 49 & 99 & & & \\
1.38 & 71 & 15 & 49.06 & 5 & 100 & & & \\
1.91 & 86 & 25 & 67.86 & 3 & 100 & & & \\
2.65 & 90 & 35 & 93.86 & 0 & 100 & & \\
3.66 & 95 & 45 & 129.83 & 0 & 100 & & \\
5.06 & 100 & 56 & 179.58 & 0 & 100 & & \\
7.00 & 95 & 66 & & & & & \\
9.69 & 90 & 76 & & & & & \\
13.40 & 52 & 82 & & & & & \\
18.54 & 55 & 88 & & & & & \\
25.64 & 52 & 94 & & & & & & \\
& & & & & & & \\
\hline
\end{tabular}

CD1 


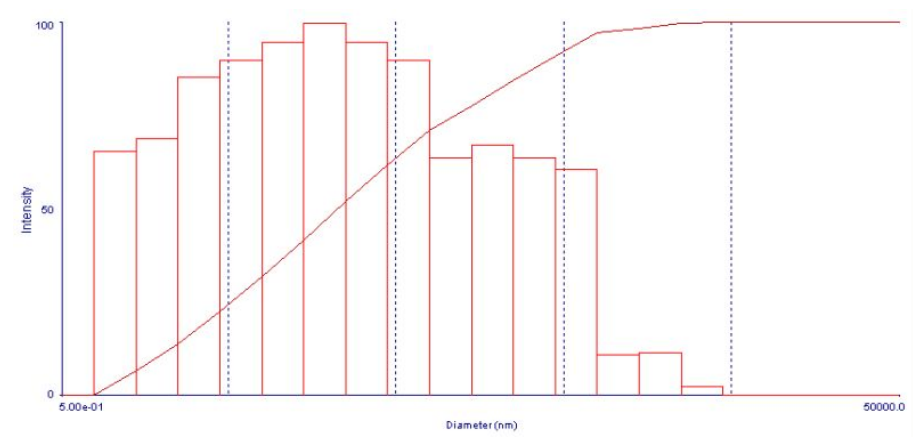

\begin{tabular}{|rrr|rrr|rrr|}
\hline$d$ & $G(d)$ & \multicolumn{1}{c}{$\mathrm{C}(\mathrm{d})$} & \multicolumn{1}{c}{$\mathrm{d}$} & $\mathrm{G}(\mathrm{d})$ & \multicolumn{1}{c|}{$\mathrm{C}(\mathrm{d})$} & $\mathrm{d}$ & $\mathrm{G}(\mathrm{d})$ & $\mathrm{C}(\mathrm{d})$ \\
\hline 1.00 & 66 & 7 & 562.34 & 61 & 97 & & & \\
1.78 & 69 & 14 & 1000.00 & 11 & 99 & & & \\
3.16 & 86 & 23 & 1778.28 & 12 & 100 & & & \\
5.62 & 90 & 32 & 3162.28 & 2 & 100 & & & \\
10.00 & 95 & 42 & 5623.41 & 0 & 100 & & & \\
17.78 & 100 & 52 & 10000.00 & 0 & 100 & & & \\
31.62 & 95 & 62 & & & & & & \\
56.23 & 90 & 71 & & & & & \\
100.00 & 64 & 78 & & & & & \\
177.83 & 67 & 85 & & & & & \\
316.23 & 64 & 91 & & & & & & \\
& & & & & & & \\
\hline
\end{tabular}

CD2

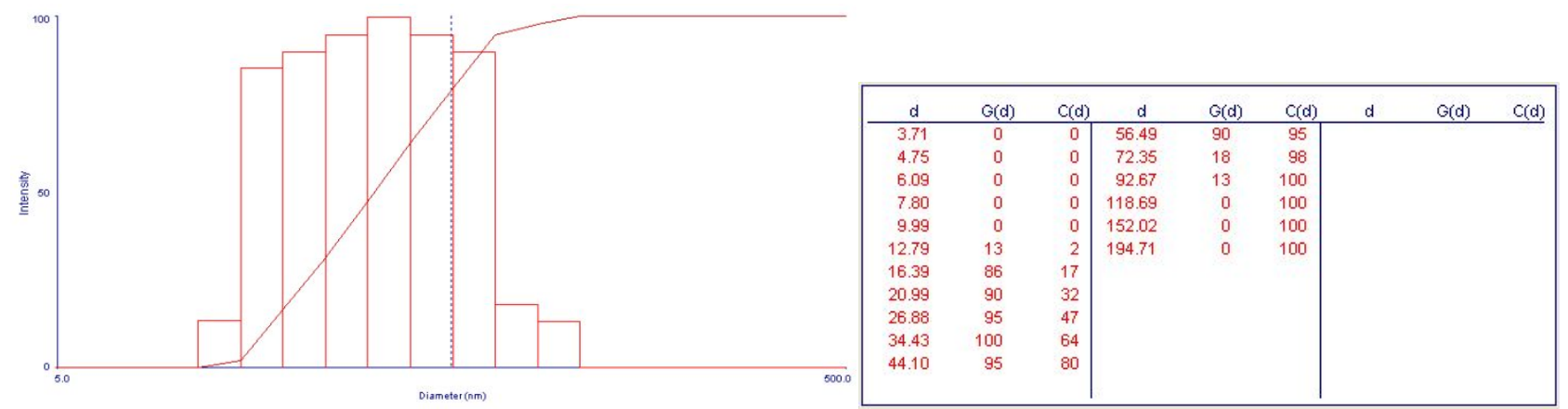

CD3 